\title{
Comparación entre Web of Science y Scopus, Estudio Bibliométrico de las Revistas de Anatomía y Morfología
}

\author{
Comparison between Web of Science and Scopus, \\ Bibliometric Study of Anatomy and Morphology Journals
}

Hernández-González, V.,"**; Sans-Rosell, N.,"***; Jové-Deltell, M. C.,*** \& Reverter-Masia, J.,***

HERNÁNDEZ-GONZÁLEZ, V. ; SANS-ROSELL, N. ; JOVÉ-DELTELL, M. C. \& REVERTER-MASIA, J. Comparación entre web of science y scopus, estudio bibliométrico de las revistas de anatomía y morfología. Int. J. Morphol., 34(4):1369-1377, 2016.

RESUMEN: El objetivo de este trabajo es comparar los indicadores bibliométricos básicos del área de anatomía y morfología dentro de las bases de datos de Web of Science (en adelante WoS) y Scopus. La metodología llevada a cabo se basa en una búsqueda bibliométrica realizada en Web of Knowledge y Scopus. La muestra la conforman el conjunto de revistas indexadas en ambas bases de datos del área citada. Los resultados indican que el número de revistas fue mayor en Scopus que en WoS (42 vs. 21). El índice citas/ artículo global es mayor en Scopus. Solamente hay dos revistas que permiten publicar sus artículos en un idioma diferente al inglés en WoS y tres en Scopus. En WoS, los valores de factor de impacto y del índice h tienen una correlación de 0,17, mientras que en la base de datos Scopus los valores de correlación entre el factor de impacto y del índice h, son muy superiores, tiene una correlación de 0,79 . La mayoría de revistas están editadas en países anglosajones. Las conclusiones del estudio nos llevan a resaltar que existe un mayor número de revistas y citas en Scopus comparado con WoS. La utilización del factor de impacto para la evaluación de una revista parece ser un indicador más conveniente a corto plazo, siendo el índice h un indicador que refleja la influencia a largo plazo de una publicación y posiblemente refleja mejor la categoría científica de ésta. En la actualidad es conveniente aconsejar el uso de varios índices en la evaluación de la calidad de las revistas.

PALABRAS CLAVE: Bibliometría; Web of Science; Scopus; Revistas científicas; Anatomía y morfología.

\section{INTRODUCCIÓN}

Hoy en día no existe duda alguna de la importancia y las ventajas de las bases de datos documentales (Manterola et al., 2014). Las bases de datos bibliográficas son la principal fuente de información utilizada en los estudios bibliométricos (Granda-Orive et al., 2013). La validez de un trabajo dependerá de la adecuada selección de la base, pues debe cubrir adecuadamente el área objeto del estudio (Granda-Orive et al.). Durante más de 40 años las bases de datos del Institute for Scientific Information (ISI) fueron las únicas que permitían el trabajo bibliométrico. Hasta no hace mucho, Web of Science [(WoS) ISI, Thomson Reuters] era la única herramienta internacional y multidisciplinaria disponible para el acceso a la literatura de ciencia, tecnología, biomedicina y otras disciplinas. A partir de esta base de datos se confecciona el Journal
Citation Report (JCR) que incluye las revistas de WoS como citantes y es donde se publica el famoso "factor de impacto" de las revistas. La WoS es una herramienta útil para la búsqueda y la alerta bibliográfica con gran número de ventajas, aunque por todos son conocidas las limitaciones que presenta así como sus inconvenientes (GrandaOrive et al.).

Para paliar estas limitaciones, a partir de 2004, Elsevier puso en marcha Scopus, una base de datos que hoy indexa 22,878 revistas peer-reviewed (SCImago Journal Rank, 2015), frente a las 13,813 de WoS (Thomson Reuters, 2015). Scopus es actualmente, por tanto, la mayor base de datos multidisciplinar existente, y ha terminado con la supremacía de WoS.

\footnotetext{
* Grupo de Investigación Movimiento Humano. Generalitat de Catalunya. Universidad de Lleida, España.

** Institut de desenvolupament social i Territorial. Universidad de Lleida.

**** Hospital Universitari Arnau de Vilanova de Lleida
} 
Como Scopus contiene también referencias bibliográficas, emplea el factor de impacto para medir la importancia de las publicaciones, su mayor cantidad de revistas fuente permite identificar a priori un mayor número de citas. Sin embargo, cabe destacar que si bien Scopus tiene mayor cobertura, su exhaustividad pierde fuerza para las referencias anteriores a 1996. Los trabajos de Bakkalbasi et al., (2006) y Bar-Ilan (2008) se han centrado en comparar los conteos de citación en ambas fuentes y concluyen que los datos de citación están fuertemente influidos por la cobertura de cada una de ellas y que la elección de una u otra depende del área temática así como del período de estudio.

Con la aparición de Scopus, aumenta la posibilidad de comparación, una característica crucial en la evaluación de la ciencia, que permite, además, los estudios complementarios que hasta ahora sólo se habían realizado sobre los datos de WoS. Varios autores han centrado sus trabajos en comparar las características de ambas bases de datos desde la perspectiva de su cobertura: títulos de revistas, áreas temáticas, idiomas, editores, distribución geográfica, etc. (López-Illescas et al., 2008; Delgado López-Cózar \& Repiso, 2013).

El índice h, que es un indicador bibliometrico para medir el impacto de las revistas, fue propuesto por Jorge Hirsch en 2005 para medir el rendimiento de un investigador, pero inmediatamente fue aplicado a las revistas (Braun et al., 2006). Una revista tiene un índice igual a $\mathrm{h}$ cuando h de sus artículos han recibido al menos h citas cada uno; es decir, una revista con índice h de 22 es aquella que ha conseguido publicar 22 artículos con al menos 22 citas cada uno de ellos. El índice h ha recibido una gran atención, dando lugar a numerosos estudios y propuestas de nuevos indicadores (Alonso et al., 2009; Egghe, 2010).

No obstante, que se tenga conocimiento, en el área de Morfología, solo se han realizado dos estudios analizando WoS y Scopus, el primero de ellos (Cantín et al., 2015a) comparando el factor de impacto (FI) y el segundo, analizando el índice $\mathrm{h}$ de los investigadores (Cantín et al., 2015b), pero nunca se ha realizado un estudio analizando el índice h de las revistas ofrecido por las dos plataformas, así como la composición de los rankings de revistas ofrecidos por estos dos productos en cuanto a la procedencia geográfica de la publicación, el idioma de edición y la editorial.

Por ello, el objetivo de este trabajo ha sido comparar entre bases de datos documentales [Web of Science (WoS) y Scopus] los indicadores bibliométricos básicos del área de Morfología y Anatomía.

\section{MATERIAL Y MÉTODO}

Para el estudio de las revistas en WoS utilizamos la fuente Journal Citation Report (JCR) en su versión Science Citation Index-Expanded, edición 2014, disponible en el portal Web of Knowledge. Para analizar las revistas de Scopus utilizamos la información disponible en el portal SCImago Journal Rank (SJR), edición de 2014, una herramienta desarrollada por SCImago Research Group, que ofrece indicadores científicos de revistas y países a partir de Scopus. Los datos sobre los indicadores de las revistas se obtuvieron a partir de las bases de datos de WoS y Scopus para el período 2010-2014.

Búsqueda bibliográfica. La búsqueda bibliográfica fue realizada en WoS a través de la plataforma ISI Web of Knowledge (Thomson Reuters) y en SCImago Research Group (Scopus,) en septiembre de 2015, en:

- WoS: Revistas indizadas en la categoría «Anatomy and Morphology» (http://ip-science.thomsonreuters.com).

- Scopus: revistas indizadas en la subcategoría «Anatomy.» (http://www.scimagojr.com/).

Se identificaron un total de 21 revistas de Anatomy and Morphology en WoS y 42 en categoría Anatomy en Scopus. Una vez localizadas las revistas se ordenaron según el factor de impacto.

Extracción de datos y variables analizadas. Para cada revista se registraron las siguientes variables: título, editorial, país, idioma de la revista, procedencia geográfica de la publicación, índice h, cuartil, número de trabajos y número de citas recibidas.

Estadística. Los datos se presentan como media $( \pm)$ desviación estándar o porcentaje. Las revistas se dividieron en cuartiles para cada uno de los índices para comparar las diferencias de clasificación. La posible correlación entre los valores de FI e índice h se analizó con la prueba de Spearman. Se considera significativo un valor de p inferior a 0,05 .

\section{RESULTADOS}

Control bibliográfico de las revistas. El número de revistas de anatomía y morfología identificadas en este estudio ha sido de 42 (Tabla I), siendo Scopus la base de datos que localiza el mayor número de revistas $(\mathrm{p}<0,001)$. Cabe destacar que existen 28 revistas en Scopus que no se encuen- 


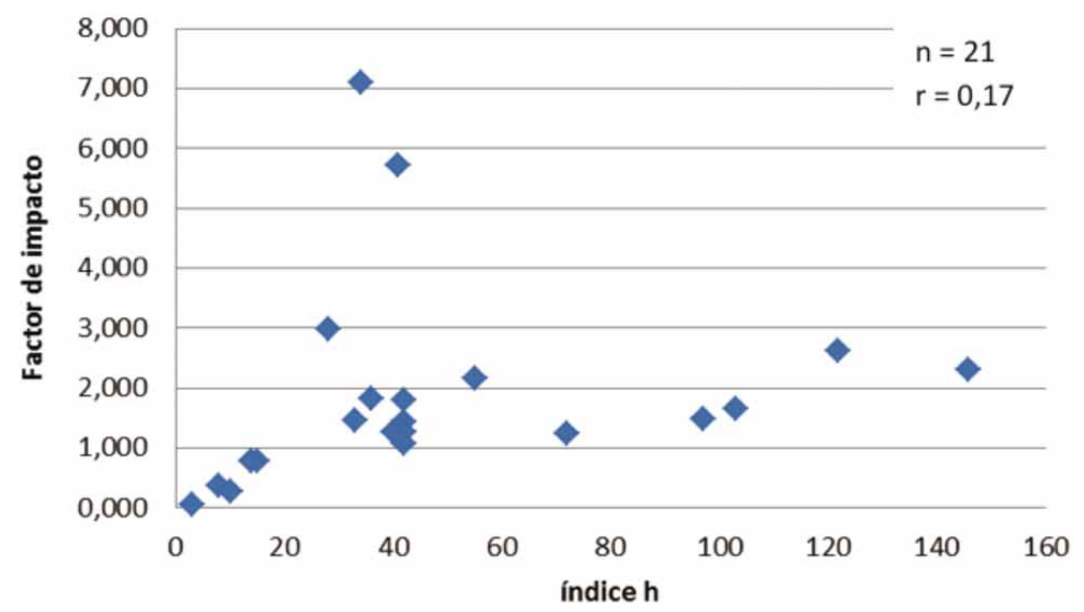

Fig. 1. Dispersión y correlación entre IF e índice h en Web of Science

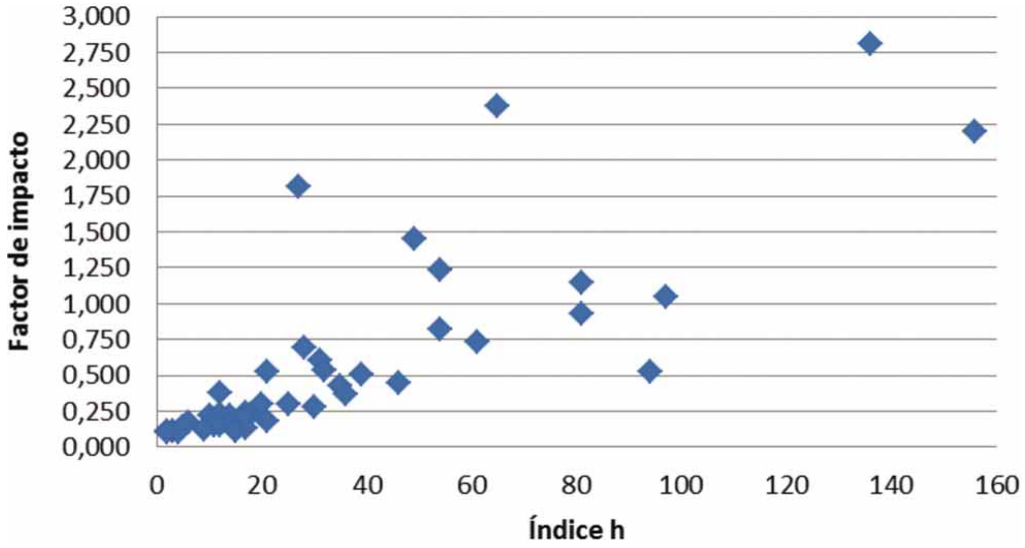

Fig. 2. Dispersión y correlación entre IF e índice h en Scopus. $n=42$ r=0,79

tran en Web of Science (Tabla I). La media anual del número de documentos fue mayor en WoS que en Scopus (102 vs 88).

En la Tabla I y II se muestra el número de citas recibidas por los artículos incluidos en el período estudiado, se observó un mayor número de citas en Scopus con respecto a WoS $(73,080$ vs 35,460).

Los artículos incluidos en el período estudiado han sido publicados en un total de 42 revistas en la base de datos Scopus, de las cuales, solo 3 publican sus artículos en varios idiomas (7,14\%) y de las 21 de WoS, solo 2 revistas publican sus artículos en varios idiomas, International Journal of Morphology y Surgical and Radiologic Anatomy (Tabla II).

Índice h. Los valores de los índices h obtenidos son altos: el $95 \%$ de las revistas de WoS, poseen un índice igual o superior a 8 , con un valor máximo de 146 y mínimo de 3, marcando diferencias acusadas entre revistas. En Scopus el $83 \%$ de las revistas tienen un índice h superior a 10, con un valor máximo de 156 y mínimo de 2.
Como se desprende de la Tabla I y II, se observa solo una moderada correspondencia entre cuartiles, índice h y FI en ambas clasificaciones. Así, por ejemplo, en WoS, las 10 revistas con mayor índice h, incluían 8 revistas que no estaban en el Q1 del FI. En WoS, los valores de FI y del índice h tenían una correlación de 0,17 , lo que indica una bajísima correlación (Fig. 1). En la base de datos Scopus los valores de correlación entre el FI y el índice h, fueron mayores, tenían una correlación de 0,79 (fig. 2). La correspondencia entre los cuartiles y el índice h en esta base de datos es mucho mayor, de las 10 revistas con mayor índice h, 8 son del primer cuartil.

Editoriales. Desde el punto de vista del tamaño, Scopus duplica en número de revistas indexadas el WoS. En WoS, el primer y segundo cuartil del índice h está dominado por revistas de origen americano (50\%) y más del $60 \%$ de las revistas están editadas por WileyBlackwell (38,09 \%) y Springer $(23,8 \%)$. En el caso de Scopus, la mitad de las revistas son de origen anglosajón y están editadas mayoritariamente por Wiley-Liss Inc. (11,6\%) y Lippincott Williams \& Wilkins $(9,3 \%)(\mathrm{Ta}-$ bla III).

Por otra parte, cabe remarcar que Scopus da más cabida a países cuya lengua vehicular no es el inglés, como son Italia, España y Brasil, cuyas revistas no figuran en WoS (Tabla III). 
Tabla I. SCImago Journal Rank (SJR). ANATOMY.

\begin{tabular}{|c|c|c|c|c|c|c|c|c|c|}
\hline Rank & Revista & País & Idioma & Editorial & $\begin{array}{l}\text { Media IF } \\
(2010-14)\end{array}$ & Cuartil & Índice $\mathrm{H}$ & $\begin{array}{l}\mathrm{N}^{\circ} \text { trabajos } \\
(2010-14)\end{array}$ & $\begin{array}{l}\text { Total citas } \\
(2010-14)\end{array}$ \\
\hline 1 & Human Brain Mapping & United States & English & Wiley-Liss Inc. & 2,808 & Q1 & 136 & 1306 & 14091 \\
\hline 2 & $\begin{array}{l}\text { American Journal of } \\
\text { Surgical Pathology }\end{array}$ & United States & English & $\begin{array}{l}\text { Lippincott Williams } \\
\text { and Wilkins. }\end{array}$ & 2,199 & Q1 & 156 & 1306 & 14605 \\
\hline 3 & $\begin{array}{l}\text { Brain Structure and } \\
\text { Function }\end{array}$ & Germany & English & Springer Verlag. & 2,372 & Q1 & 65 & 732 & 4717 \\
\hline 4 & $\begin{array}{c}\text { Frontiers in } \\
\text { Neuroanatomy } \\
\text { Advances in Anatomy }\end{array}$ & Switzerland & English & $\begin{array}{l}\text { Frontiers Research } \\
\text { Foundation }\end{array}$ & 1,819 & Q1 & 27 & 373 & 2301 \\
\hline 5 & $\begin{array}{c}\text { Embryology and Cell } \\
\text { Biology }\end{array}$ & Germany & English & Springer Verlag. & 0,693 & Q1 & 28 & 29 & 161 \\
\hline 6 & Brain Topography & United States & English & $\begin{array}{c}\text { Kluwer } \\
\text { Academic/Human } \\
\text { Sciences Press Inc.. }\end{array}$ & 1,449 & Q1 & 49 & 312 & 889 \\
\hline 7 & $\begin{array}{l}\text { Advances in Anatomic } \\
\text { Pathology }\end{array}$ & United States & English & $\begin{array}{l}\text { Lippincott Williams } \\
\text { and Wilkins. }\end{array}$ & 1,236 & Q1 & 54 & 228 & 1761 \\
\hline 8 & $\begin{array}{l}\text { Journal of Histochemistry } \\
\text { and Cytochemistry }\end{array}$ & United States & English & $\begin{array}{l}\text { Histochemical } \\
\text { Society Inc. }\end{array}$ & 1,048 & Q1 & 97 & 420 & 2637 \\
\hline 9 & $\begin{array}{l}\text { American Journal of } \\
\text { Physical Anthropology }\end{array}$ & United States & English & Wiley-Liss Inc. & 1,148 & Q1 & 81 & 1050 & 5403 \\
\hline 10 & $\begin{array}{c}\text { American Journal of } \\
\text { Human Biology }\end{array}$ & United States & English & Wiley-Liss Inc. & 0,822 & Q1 & 54 & 587 & 2666 \\
\hline 11 & Journal of Anatomy & $\begin{array}{l}\text { United } \\
\text { Kingdom }\end{array}$ & English & Wiley-Blackwell. & 0,928 & Q1 & 81 & 642 & 3533 \\
\hline 12 & Cells Tissues Organs & Switzerland & English & S. Karger AG. & 0,735 & Q2 & 61 & 399 & 1314 \\
\hline 13 & A natomical Record & United States & English & $\begin{array}{l}\text { John Wiley and } \\
\text { Sons Inc.. }\end{array}$ & 0,602 & Q2 & 31 & 1104 & 3886 \\
\hline 14 & Annals of Anatomy & Germany & English & $\begin{array}{l}\text { Urban und Fischer } \\
\text { Verlag Jena. }\end{array}$ & 0,532 & Q2 & 32 & 368 & 1540 \\
\hline 15 & $\begin{array}{l}\text { Surgical and Radiologic } \\
\text { Anatomy }\end{array}$ & France & $\begin{array}{c}\text { Multi- } \\
\text { languages }\end{array}$ & Springer Paris. & 0,506 & Q2 & 39 & 865 & 1157 \\
\hline 16 & $\begin{array}{c}\text { Anatomical Sciences } \\
\text { Education }\end{array}$ & United States & English & $\begin{array}{l}\text { John Wiley and } \\
\text { Sons Inc.. }\end{array}$ & 0,527 & Q2 & 21 & 327 & 1420 \\
\hline 17 & Cryo-Letters & $\begin{array}{l}\text { United } \\
\text { Kingdom }\end{array}$ & English & Cryo-Letters. & 0,430 & Q2 & 35 & 291 & 587 \\
\hline 18 & $\begin{array}{l}\text { Microscopy Research and } \\
\text { Technique }\end{array}$ & United States & English & Wiley-Liss Inc. & 0,524 & Q2 & 94 & 806 & 2550 \\
\hline 19 & Clinical Anatomy & United States & English & Wiley-Liss Inc. & 0,448 & Q2 & 46 & 838 & 2200 \\
\hline 20 & $\begin{array}{l}\text { International Journal of } \\
\text { Surgical Pathology }\end{array}$ & United States & English & $\begin{array}{l}\text { SAGE Publications } \\
\text { Inc. } \\
\text { Hacettepe }\end{array}$ & 0,368 & Q2 & 36 & 709 & 1401 \\
\hline 21 & Neuroanatomy & Turkey & English & $\begin{array}{l}\text { University Faculty } \\
\text { of Medici. }\end{array}$ & 0,2168 & Q2 & 10 & 3 & 9 \\
\hline 22 & Clinical Dysmorphology & $\begin{array}{l}\text { United } \\
\text { Kingdom }\end{array}$ & English & $\begin{array}{l}\text { Lippincott Williams } \\
\text { and Wilkins. }\end{array}$ & 0,2954 & Q3 & 20 & 204 & 151 \\
\hline 23 & Arte ry Research & Netherlands & English & Elsevier BV. & 0,3804 & Q3 & 12 & 118 & 237 \\
\hline 24 & $\begin{array}{l}\text { Anatomical Science } \\
\text { International }\end{array}$ & United States & English & $\begin{array}{c}\text { American } \\
\text { Orthopsychiatric } \\
\text { Association Inc.. } \\
\text { Okajima Foria }\end{array}$ & 0,3006 & Q3 & 25 & 224 & 332 \\
\hline 25 & $\begin{array}{c}\text { Okajimas Folia } \\
\text { Anatomica Japonica }\end{array}$ & Japan & English & $\begin{array}{l}\text { Anatomica } \\
\text { Yaponika }\end{array}$ & 0,2216 & Q3 & 14 & 106 & 114 \\
\hline 26 & $\begin{array}{l}\text { International Journal of } \\
\text { Morphology }\end{array}$ & Chile & $\begin{array}{c}\text { Multi- } \\
\text { language }\end{array}$ & $\begin{array}{l}\text { Henshubu. } \\
\text { Universidad de la } \\
\text { Frontera. }\end{array}$ & 0,2276 & Q3 & 12 & 1174 & 960 \\
\hline 27 & $\begin{array}{l}\text { Journal of Clinical } \\
\text { Dysmophology }\end{array}$ & United States & English & $\begin{array}{l}\text { Lippincott Williams } \\
\text { and Wilkins. }\end{array}$ & 0,2342 & Q3 & 18 & 186 & 151 \\
\hline 28 & Folia Morphologica & Poland & English & Via Medica & 0,2378 & Q3 & 17 & 299 & 367 \\
\hline 29 & $\begin{array}{c}\text { European Journal of } \\
\text { Anatomy }\end{array}$ & Spain & English & $\begin{array}{l}\text { Sociedad Anatomica } \\
\text { Espanola. }\end{array}$ & 0,1526 & Q3 & 12 & 171 & 71 \\
\hline 30 & $\begin{array}{c}\text { Analytical and } \\
\text { Quantitative Cytology } \\
\text { and Histology }\end{array}$ & United States & English & $\begin{array}{l}\text { Science Printers and } \\
\text { Publishers Inc.. }\end{array}$ & 0,283 & Q3 & 30 & 237 & 300 \\
\hline 31 & $\begin{array}{c}\text { Comparative Clinical } \\
\text { Pathology }\end{array}$ & $\begin{array}{l}\text { United } \\
\text { Kingdom }\end{array}$ & English & Springer London & 0,2146 & Q3 & 17 & 1188 & 846 \\
\hline 32 & $\begin{array}{l}\text { Italian Joumal of } \\
\text { Anatomy and } \\
\text { Embrvology }\end{array}$ & Italy & English & $\begin{array}{c}\text { Firenze University } \\
\text { Press. }\end{array}$ & 0,186 & Q3 & 21 & 249 & 230 \\
\hline
\end{tabular}




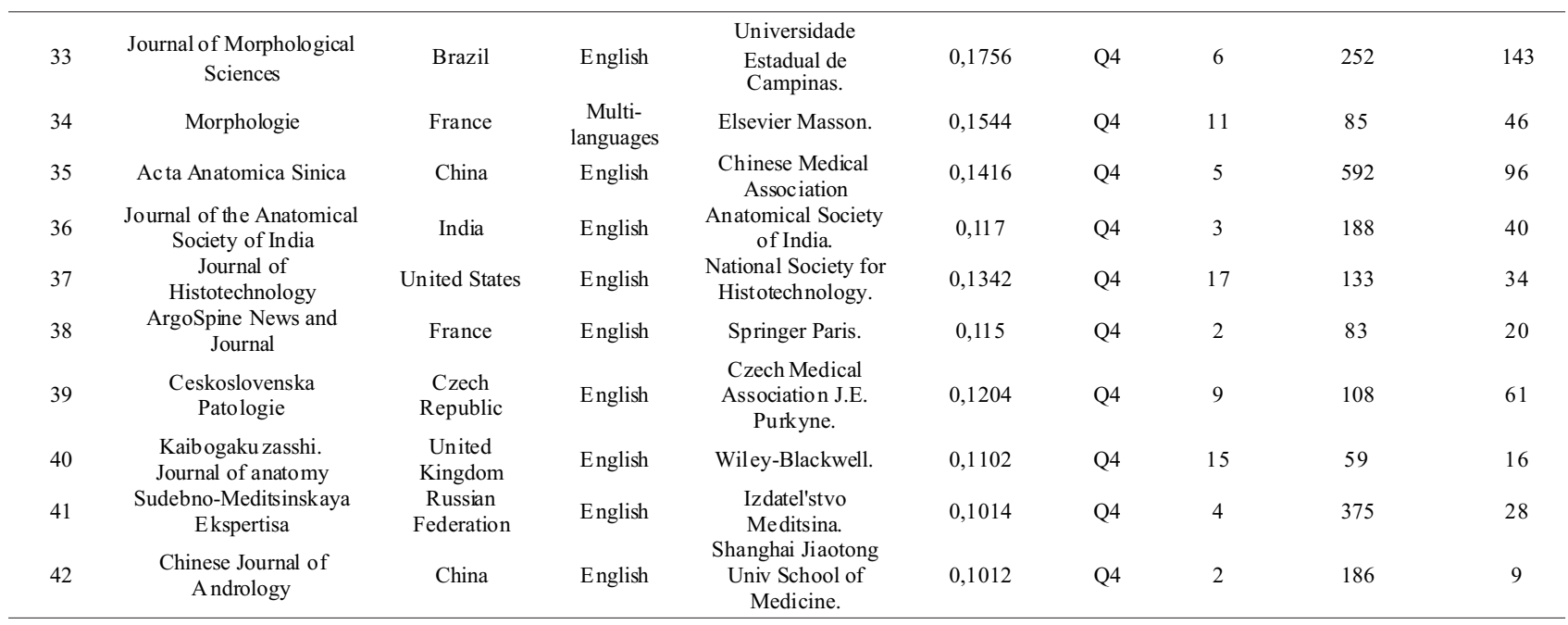

Table II. Sciences Citation Index (SCI). ANATOMY AND MORPHOLOGY

\begin{tabular}{|c|c|c|c|c|c|}
\hline Web of Science & $\begin{array}{l}\mathrm{N}^{\mathrm{o}} \\
\text { registros }\end{array}$ & $\%$ & Scopus & $\begin{array}{l}\mathrm{N}^{\mathrm{o}} \\
\text { registros }\end{array}$ & $\%$ \\
\hline Wiley-Blackwell & 8 & 38,09 & Wiley-Liss Inc. & 5 & 11,62 \\
\hline Springer & 2 & 9,52 & LIPPINCOTT WILLIAMS \& & 4 & 9,3 \\
\hline Springer France & 1 & 4,76 & John Wiley and Sons Inc.. & 3 & 6,97 \\
\hline Springer Heidelberg & 1 & 4,76 & WILEY-BLACKWELL & 2 & 4,65 \\
\hline Springer-Verlag Berlin & 1 & 4,76 & Springer Paris. & 2 & 4,65 \\
\hline Anatomical Soc India & 1 & 4,76 & SPRINGER-VERLAG & 2 & 4,65 \\
\hline Churchill Livingstone & 1 & 4,76 & American Orthopsychiatric Association & 1 & 2,32 \\
\hline Elsevier Gmbh, Urban \& & 1 & 4,76 & ANATOMICAL SOC INDIA & 1 & 2,32 \\
\hline Frontiers Research Foundation & 1 & 4,76 & Chinese Medical Association & 1 & 2,32 \\
\hline Karger & 1 & 4,76 & Cryo-Letters. & 1 & 2,32 \\
\hline Lippincott Williams \& Wilkins & 1 & 4,76 & Czech Medical Association J.E. & 1 & 2,32 \\
\hline Soc Chilena Anatomia & 1 & 4,76 & Elsevier BV. & 1 & 2,32 \\
\hline \multirow{19}{*}{ Via Medica } & 1 & 4,76 & Elsevier Masson. & 1 & 2,32 \\
\hline & & & Firenze University Press. & 1 & 2,32 \\
\hline & & & FRONTIERS RESEARCH & 1 & 2,32 \\
\hline & & & Hacettepe University Faculty of & 1 & 2,32 \\
\hline & & & Histochemical Society Inc. & 1 & 2,32 \\
\hline & & & Izdatel'stvo Meditsina. & 1 & 2,32 \\
\hline & & & Kluwer Academic/Human Sciences & 1 & 2,32 \\
\hline & & & National Society for Histotechnology. & 1 & 2,32 \\
\hline & & & Okajima Foria Anatomica Yaponika & 1 & 2,32 \\
\hline & & & S. Karger AG. & 1 & 2,32 \\
\hline & & & SAGE Publications Inc.. & 1 & 2,32 \\
\hline & & & Science Printers and Publishers Inc.. & 1 & 2,32 \\
\hline & & & Shanghai Jiaotong University School of & 1 & 2,32 \\
\hline & & & Sbeedeciand Anatomica Espanola. & 1 & 2,32 \\
\hline & & & Springer London & 1 & 2,32 \\
\hline & & & Universidad de la Frontera. & 1 & 2,32 \\
\hline & & & Universidade Estadual de Campinas. & 1 & 2,32 \\
\hline & & & Urban und Fischer Verlag Jena. & 1 & 2,32 \\
\hline & & & VIA MEDICA & 1 & 2,32 \\
\hline
\end{tabular}


Tabla III. Número de revistas y porcentajes de publicaciones por editoriales en el período 2010-2014

\begin{tabular}{|c|c|c|c|c|c|}
\hline Web of Science & $\begin{array}{l}\mathrm{N}^{\mathrm{o}} \\
\text { registros }\end{array}$ & $\%$ & Scopus & $\begin{array}{l}\mathrm{N}^{\mathrm{o}} \\
\text { registros }\end{array}$ & $\%$ \\
\hline Wiley-Blackwell & 8 & 38,09 & Wiley-Liss Inc. & 5 & 11,62 \\
\hline Springer & 2 & 9,52 & LIPPINCOTT WILLIAMS \& WILKINS & 4 & 9,3 \\
\hline Springer France & 1 & 4,76 & John Wiley and Sons Inc. & 3 & 6,97 \\
\hline Springer He idelberg & 1 & 4,76 & WILEY-BLACKWELL & 2 & 4,65 \\
\hline Springer-Verlag Berlin & 1 & 4,76 & Springer Paris. & 2 & 4,65 \\
\hline Anatomical Soc India & 1 & 4,76 & SPRINGER-VERLAG & 2 & 4,65 \\
\hline Churchill Livingstone & 1 & 4,76 & American Orthopsychiatric Association & 1 & 2,32 \\
\hline Elsevier Gmbh, Urban \& & 1 & 4,76 & ANATOMICAL SOC INDIA & 1 & 2,32 \\
\hline Frontiers Research Foundation & 1 & 4,76 & Chinese Medical Association & 1 & 2,32 \\
\hline Karger & 1 & 4,76 & Cryo-Letters. & 1 & 2,32 \\
\hline Lippincott Williams \& Wilkins & 1 & 4,76 & Czech Medical Association J.E. Purkyne. & 1 & 2,32 \\
\hline Soc Chilena Anatomia & 1 & 4,76 & Elsevier BV. & 1 & 2,32 \\
\hline \multirow[t]{19}{*}{ Via Medica } & 1 & 4,76 & Elsevier Masson. & 1 & 2,32 \\
\hline & & & Firenze University Press. & 1 & 2,32 \\
\hline & & & FRONTIERS RESEARCH & 1 & 2,32 \\
\hline & & & Hacettepe University Faculty of Medicine. & 1 & 2,32 \\
\hline & & & Histochemical Society Inc. & 1 & 2,32 \\
\hline & & & Izdatel'stvo Meditsina. & 1 & 2,32 \\
\hline & & & Kluwer Academic/Human Sciences Press & 1 & 2,32 \\
\hline & & & National Society for Histotechnology. & 1 & 2,32 \\
\hline & & & Okajima Foria Anatomica Yaponika & 1 & 2,32 \\
\hline & & & S. Karger AG. & 1 & 2,32 \\
\hline & & & SAGE Publications Inc.. & 1 & 2,32 \\
\hline & & & Science Printers and Publishers Inc. & 1 & 2,32 \\
\hline & & & Shanghai Jiaotong University School of & 1 & 2,32 \\
\hline & & & Socie dad Anatomica Espanola. & 1 & 2,32 \\
\hline & & & Springer London & 1 & 2,32 \\
\hline & & & Universidad de la Frontera. & 1 & 2,32 \\
\hline & & & Universidade Estadual de Campinas. & 1 & 2,32 \\
\hline & & & Urban und Fischer Verlag Jena. & 1 & 2,32 \\
\hline & & & VIA MEDICA & 1 & 2,32 \\
\hline
\end{tabular}

\section{DISCUSIÓN}

Hemos podido comprobar, que en las áreas de anatomía y morfología existe un mayor número de revistas en Scopus al compararlo con WoS, con diferencias significativas a favor de Scopus. En otro análisis, análogo en otra área de conocimiento, López-Illescas et al., al analizar la cobertura y el impacto en ambas bases de datos sobre revistas en oncología, observaron resultados similares. Estos autores afirman que estas diferencias son debidas a una mayor cobertura de Scopus en países no anglo parlantes, hecho que se aprecia, también, en los resultados de nuestro estudio. Del análisis realizado, se observa que hay una representación de países mucho mayor en Scopus, no obstante, resulta curioso que habiendo representación de países como Brasil, España o Italia, solo haya dos revistas que publiquen artículos además en dos idiomas, la Int. J. Morphol. en español e inglés y y la
Morphologie que lo hace en francés e inglés, sucediendo lo mismo en WoS con Int. J. Morphol. y Morphologie.

Al respecto, señalar que, tal y como afirma Quindós (2009) se debería hacer una discriminación positiva de todos aquellos artículos escritos en lenguas científicas minoritarias que reciben un número no desdeñable de citaciones bibliográficas y más concretamente en Español. Este mismo autor argumenta que aunque el inglés sea la lengua franca de la ciencia actual, no se debería tolerar que esta lengua sirva de pretexto y permita que los países anglosajones hayan creado un monopolio de la comunicación científica.

En este estudio se aprecia que una amplia mayoría de revistas están editadas por una misma empresa y/o institución, y esta es anglosajona. Quindós, argumenta que algo tan aleatorio como es el lugar o la cultura donde se ha nacido o crecido, no debería beneficiar a unos en perjuicio de 
otros cuando la calidad científica es incuestionable. Las autoridades científicas de un país deberían potenciar la publicación científica de calidad en la lengua oficial del propio país, algo que no ocurre en las revistas analizadas, ya que, por ejemplo, European Journal of Anatomy (Española) o Journal of Morphological Sciences (Brasil) publican sus artículos en inglés. En esta misma línea Aréchaga (2011) manifiesta que los propios culpables de la no potenciación de las revistas iberoamericanas son los propios investigadores de un país, que desdeñan las revistas nacionales e intentan publicar los mejores trabajos fuera, dejando aquellos de menor calidad para consumo interno.

En la comunidad científica iberoamericana se da la paradoja que donde los gobiernos subvencionan las publicaciones locales, de manera simultánea llevan a cabo una acción de minusvalorar los artículos que se publican en esas mismas revistas que subvencionan (Ugarte, 2004). Aréchaga y Quindós, afirman que debemos resolver esta paradoja y promover una valoración más positiva de nuestras revistas para conseguir un efecto que ayude a la investigación de nuestras naciones con la publicación de mejores contribuciones científicas. Un apoyo efectivo a las revistas científicas irá unido, de forma indisociable, al incremento de la calidad de los trabajos de investigación y esta puede ser una fuente importante de generación de riqueza para nuestro país (Aréchaga).

Tal como han apuntado diferentes estudios (Glänzel et al., 2006 \& Buela-Casal, 2010) la ciencia en América Latina ha experimentado un vertiginoso incremento en los últimos años en términos de publicaciones científicas en las bases de datos de WoS, sin embargo, tal y como se aprecia en este trabajo, en el campo de la morfología y anatomía todavía queda mucho trabajo por realizar, ya que sólo existe una revista chilena indexada en WoS que publica en español y en inglés. Probablemente, se deba al criterio de selección de revistas de WoS, que se basa en varios indicadores como son: que la revista publique, al menos, tres números al año consecutivos con revisión por pares; es preciso indicar las direcciones de la editorial y del editor y enviar una carta de explicación sobre que aporta la revista en cuestión en su área de especialización o cobertura. Posteriormente, la entrada en el JCR, se basa en la noción de la utilización del factor de impacto para medir la importancia de las revistas científicas en el sistema internacional de comunicación.

Manterola et al. (2005), en un estudio realizado en revistas quirúrgicas, sugieren que el FI puede considerarse un indicador de calidad metodológica razonable para la evaluación de artículos científicos. No obstante, tal y como afirma Cantin et al., (2015a), todas las revistas indexadas en WoS y Scopus tienen un alto nivel de calidad, sin embargo la cobertura de Scopus resulta más amplia y menos estricta, siendo el impacto de citación de las revistas menos discriminativo. Este hecho también explicaría que, en nuestro trabajo en Scopus, haya el doble de revistas indexadas que en WoS.

El FI es o debería ser el índice más utilizado por los investigadores para evaluar a las revistas de anatomía y morfología (Cantín et al., 2015a), pero sus deficiencias y limitaciones deben ser tomadas en consideración cuando se utilice por sí solo. Por esta razón, Cantín et al. (2015b), propone la utilización del índice h como una herramienta fiable para cuantificar la productividad académica dentro de la morfología, fácilmente calculable, y puede ser útil en la evaluación de las decisiones relativas a la promoción, dentro de los departamentos académicos, en el área morfológica.

En nuestro estudio, los datos del FI y el índice h de las revistas de morfología y anatomía incluidas en una misma base de datos ofrecen una información bibliométrica distinta; la correlación en WoS es bajísima y en Scopus aceptable. El FI analiza las citas a corto plazo y el índice h incluye la trayectoria de la revista a más largo plazo. Como se aprecia en nuestros resultados, no son las mismas revistas las que tienen mayor impacto a corto y a largo plazo. El largo plazo parece reflejar mejor la influencia de esa revista en la comunidad científica. Por ello, aunque el uso del FI está muy extendido, el índice h podría ser un indicador mucho más preciso de la calidad de las revistas científicas (Grupo Scimago, 2006).

Lógicamente, como cualquier parámetro bibliométrico, el Índice $\mathrm{h}$ también tiene limitaciones (Aguayo-Albasini \& Campillo-Soto, 2008); tendrán menos citas las áreas muy específicas y con menor número de grupos de investigación. Diversos autores han señalado que el Índice h, tomado de modo absoluto, no puede ser usado para comparar la investigación de investigadores de ciencias diferentes (Costas \& Bordons, 2007). Por ejemplo, Hirsch mostró que la h promedio en física era de 45, mientras que en ciencias de la vida era casi del doble. De acuerdo con Glänzel \& Persson (2005), entre los ganadores de la Medalla Price, que premia a los que más contribuciones han aportado a la cienciometría (bibliometría), Eugene Garfield tenía un Índice h de 16, Tibor Braun de 17, Henry Small de 9, van Raan de 16, Ben Martin de 11, Francis Narin de 16, András Schubert de 17, Glänzel de 17, Moed de 15, Leydesdorff de 13, Egghe de 12, Rousseau de 11, Ingwersen de 10, y White de 10 (Iglesias \& Pecharromán, 2006). En relación al índice h de revistas parece ocurrir lo mismo. Garcia-Pachón \& Padilla-Navas (2014), en revistas biomédicas, observaron una disparidad de un Índice h de 2 a 62. En nuestro trabajo ocurre exactamente lo mismo. 
En el área morfológica Cantín et al. (2015b), afirmó que el Índice $\mathrm{h}$ de los investigadores es una herramienta fiable para cuantificar la productividad académica dentro de la morfología, fácilmente calculable y puede ser útil en la evaluación de las decisiones relativas a la promoción dentro de los departamentos académicos. Nuestro estudio complementaria el anterior y confirmaría que el índice h de las revistas también es una herramienta fiable y útil para la evaluación de la producción científica.

Un aspecto relevante de nuestro trabajo ha sido que, aunque la media total de artículos por base de datos es mayor en WoS, el índice citas/artículo global es mayor en Scopus. La mayor cantidad de revistas de Scopus seguramente propicia una mayor citación de los trabajos. Estos confirmarían los trabajos realizados por otros autores en áreas de conocimiento diferentes (Bakkalbasi et al.; Neuhaus \& Daniel, 2008; Bar-Ilan), donde concluyen que los datos de citación están fuertemente influidos por la cobertura de la base de datos empleada.
En este trabajo hemos tratado de analizar las revistas científicas del área de anatomía y morfología. Las dos bases de datos estudiadas presentan comportamientos diferentes en algunos indicadores, no obstante, parece conveniente aconsejar el uso de varias bases de datos en la evaluación de la calidad de las revistas. Por otra parte, sería recomendable que las revistas científicas de anatomía y morfología de países latinoamericanos realizaran un esfuerzo en ser incluidas en las dos bases de datos analizadas; de esta manera se contribuiría a dar mayor visibilidad e impacto a las publicaciones realizadas en lenguas no anglosajonas.

\section{AGRADECIMIENTOS}

Este trabajo fue apoyado financieramente por el proyecto «Identificación de competències docentes, investigadoras y de gestión del profesorado universitario $(n<0821)$ Universidad de Lleida y el Proyecto Movimiento Humano (SGR 917) Generalitat de Cataluña, España».

HERNÁNDEZ-GONZÁLEZ, V. ; SANS-ROSELL, N. ; JOVÉ-DELTELL, M. C. \& REVERTER-MASIA, J. Comparison between web of science and scopus, bibliometric study of anatomy and morphology journals. Int. J. Morphol., 34(4):1369-1377, 2016.

SUMMARY: The aim of this study is to compare the basic bibliometric indicators in the area of anatomy and morphology inside the databases Web of Science and Scopus. The methodology carried out is based on a bibliometric search conducted in Scopus and Web of Knowledge. The sample comprises the set of journals indexed in both databases of the cited field. The results indicate that the number of journals was higher in Scopus than WoS (42 vs. 21). The overall reference / article index is higher in Scopus. There are only two journals that allow publication of papers in a language other than English, one in WoS and three in Scopus. In WoS, the impact factor value and h-index has a correlation of 0.17 , while the Scopus database correlation values between the impact factor and h-index, are far superior, it has a correlation 0.79. Most journals are published in English-speaking countries. These findings lead us to highlight that there are a greater number of citations in Scopus journals compared with WoS. The use of the impact factor to evaluate a journal seems to be a more convenient short-term indicator, the $\mathrm{h}$ index is an indicator that reflects the long-term influence of a publication and could also better determine the article's scientific category. Currently, the use of various indices is advisable in evaluating the quality of journals.

KEY WORDS: Bibliometrics; Web of Science; Scopus; Scientific journals; Anatomy and morphology.

\section{REFERENCIAS BIBLIOGRÁFICAS}

Alonso, S.; Cabrerizo, F. J.; Herrera-Viedma, E. \& Herrera, F. $\mathrm{H}$-index: A review focused in its variants, computation and standardization for different scientific field. J. Informetr., 3(4):273-89, 2009.

Aréchaga, J. El prestigio y la rentabilidad de las revistas científicas españolas se basa en el uso internacional de sus contenidos. Endocrinol. Nutr., 58(2):57-61, 2011.

Aguayo-Albasini, J. L. \& Campillo-Soto, A. Evaluación de la actividad investigadora mediante el índice h de Hirsch. Med. Clin., 131(6):239, 2008.
Bakkalbasi, N.; Bauer, K.; Glover, J. \& Wang, L. Three options for citation tracking: Google Scholar, Scopus and Web of Science. Biomed. Digit. Libr., 3:7, 2006.

Bar-Ilan, J. Which h-index? - A comparison of WoS, Scopus and Google Scholar. Scientometrics, 74(2):257-71, 2008.

Braun, T.; Glänzel, W. \& Schubert, A. A Hirsch-type index fort journals. Scientometrics, 69(1):169-73, 2006.

Cantín, M.; Muñoz, M. \& Roa, I. Comparison between Impact Factor, Eigenfactor Score, and SCImago journal rank indicator 
in anatomy and morphology journals. Int. J. Morphol., $33(3): 1183-8,2015 \mathrm{a}$

Cantín, M.; Muñoz, M. \& Roa, I. The h-index in academic morphology. Int. J. Morphol., 33(2):706-11, 2015 b.

Costas, R. \& Bordons, M. Una visión crítica del índice h: algunas consideraciones derivadas de su aplicación práctica. Prof. Inf., 16(5):427-32, 2007.

Delgado López-Cózar, E. \& Repiso, R. El impacto de las revistas de comunicación: comparando Google Scholar Metrics, Web of Science y Scopus. Comunicar, 21(41):45-52, 2013.

Egghe, L. The Hirsch index and related impact measures. Ann. Rev. Inf. Sci. Technol., 44(1):65-114, 2010.

Garcia-Pachón, E. \& Padilla-Navas, I. El factor de impacto y el índice h de las revistas biomédicas españolas. Med. Clin. (Barc.), 142(5):226-7, 2014.

Glänzel, W. \& Persson, O. H-index for Price medalists. ISSI Newsl., 1(4):15-8, 2005.

Glänzel, W.; Leta, J. \& Thijs, B. Science in Brazil. Part 1: A macrolevel comparative study. Scientometrics, 67(1):67-86, 2006.

Granda-Orive, J. I.; Alonso-Arroyo, A.; García-Río, F.; SolanoReina, S.; Jiménez-Ruiz, C. A. \& Aleixandre-Benavent, R. Ciertas ventajas de Scopus sobre Web of Science en un análisis bibliométrico sobre tabaquismo. Rev. Esp. Doc. Cient., 36(2):1-9, 2013.

Grupo Scimago. El índice h de Hirsch: aportaciones a un debate. Prof. Inf., 15(4):304-6, 2006.

Iglesias, J. E. \& Pecharromán, C. Scaling the h-index for different scientific fields. arXiv.org, Cornell University Library, 2006. Available from: http://arxiv.org/ftp/physics/papers/0607/ 0607224.pdf

López-Illescas, C.; de Moya-Anegón, F. \& Moed, H. F. Coverage and citation impact of oncological journals in the Web of Science and Scopus. J. Informetr., 2(4):304-16, 2008.

Manterola, C.; Pineda, V.; Vial, M. \& Losada, H. ¿Es el factor de impacto un índice apropiado para determinar el grado de evidencia de estudios sobre procedimientos terapéuticos en revistas quirúrgicas? Cir. Esp., 78(2):96-9, 2005.

Manterola, C.; Otzen, T. \& Calo, L. Electronic resources for searching health scientific information. Database publication type. Int. J. Morphol., 32(4):1484-91, 2014.

Neuhaus, C. \& Daniel, H. D. Data sources for performing citation analysis: An overview. J. Doc., 64(2):193-210, 2008.

Quindós, G. Confundiendo al confuso: reflexiones sobre el factor de impacto, el índice h(irsch), el valor Q y otros cofactores que influyen en la felicidad del investigado. Rev. Iberoam. Micol., 26(2):97-102, 2009.

Ugarte P., F. La edición de revistas científicas en Latinoamérica. Rev. Chil. Pediatr., 75(6):509-11, 2004.

Zych, I. \& Buela-Casal, G. Internacionalidad de las revistas de psicología multidisciplinar editadas en iberoamérica e incluidas en la Web of Science. Univ. Psychol., 9(1):27-34, 2010.

Dirección para Correspondencia:

Dr. Joaquín Reverter Masia

Grupo de Investigación Movimiento Humano

Universidad de Lleida

ESPAÑA

Email: reverter@didesp.udl.cat

Received: 18-11-2015

Accepted: 06-09-2016 